\title{
Polymorphic eruption of pregnancy - new therapeutic approach
}

\author{
Martina Part ${ }^{1 *}$, Barbora Gulanova ${ }^{2}$ and Maria Simaljakova ${ }^{3}$ \\ ${ }^{1}$ Faculty of Medicine, Comenius University in Bratislava, Dept. of Dermatology and Venerology, Bratislava, Slovakia \\ ${ }^{2}$ University Hospital, Dept. of Dermatology and Venerology, Bratislava, Slovakia \\ ${ }^{3}$ Faculty of Medicine, Comenius University in Bratislava, Dept. of Dermatology and Venerology, Bratislava, Slovakia
}

\begin{abstract}
Background: Polymorphic eruption of pregnancy (PEP) also known as pruritic urticarial papules and plaques of pregnancy (PUPPP) is a benign disease associated with pregnancy. The polymorphic lesions usually begin to appear in the abdominal striae with sparing of periumbilical area. Etiology of this condition is unknown.
\end{abstract}

Methods: Women with polymorphic eruption of pregnancy were randomized into two groups; Group A was treated with systemic corticosteroids and antihistamines and group B was treated with autologous haemotherapy. The efficacy of different therapeutic options was assessed.

Results: In our study, we enrolled 6 women, In Group A ( $\mathrm{n}=3 / 50 \%)$ the efficacy of therapy was very low. All participants took systemic corticosteroids and antihistamines until delivery. The skin changes were stable, patients suffered from serious pruritus. In Group B ( $n=3 / 50 \%$ ) we used intramuscular autologous haemotherapy with whole blood in weekly intervals. Generally, pruritus improved in 2 days and skin changes disappeared in 10-15 days after starting the therapy.

Conclusions: According to our study, intramuscular autologous haemotherapy should be considered as an effective alternative therapeutic method with a very good efficacy and safety profile for the mother as well as the child.

\section{Introduction}

During pregnancy, women may develop a lot of changes, including specific skin changes. Many of these are accepted as a physiological changes in pregnancy, but some of them may be more serious with different maternal and fetal risks.

Polymorphic eruption of pregnancy aslo known as pruritic urticarial papules and plaques of pregnancy is a benign disease associated with pregnancy. Patients suffering from this disorder represent a heterogenous group of intensely pruritic dermatoses with variable clinical eruptions (erythematous macules and papules, urticarial plaques, even vesicles or targetoid lesions). Polymorphic rash usually occurs in abdominal striae, with sparing of the umbilical area [1]. Lesions may gradually involve the trunk and extremities. The palms, soles and face are usually not affected. Despite the severe pruritus, lack of excoriations on the skin is present. PEP has no specific marker in laboratory parameters or in histological features [2]. The histological examination revealed nonspecific features such as dermal lymphocytic perivascular infiltrate or spongiosis, immunoflorescence is also negative. PEP is a self-limited disorder with spontaneous regression within 6 weeks after delivery [3]. Only some cases of PEP presenting in postpartum period are refered [2]. PEP has an incidence about 1:200 pregnant women [1]. The disease usually occurres in primigravidas in the third trimester (most frequently in $35^{\text {th }}$ to $37^{\text {th }}$ week), in multiple gestation pregnancies, or in pregnancies with male babies. Recurrency in subsequent pregnancies is extremely rare [4]. In a case of recurrence, the rash is less severe. In general, the prognosis of PEP has no maternal or fetal risk.

\section{Material and methods}

Six pregnant women with PEP were enrolled to our study. For the purpose of this study, PEP was defined at the presence of pruritic dermatosis with polymorphic rash (erythematous macules and papules, urticarial plaques, targetoid lesions) that started during the second third of the pregnancy. Other causes of exanthema (not specific for pregnancy) were excluded with serological and histological examination. Skin biopsies from lesions were non-specific, as well as when tested with direct immunoflorescence. Women fulfilled this criteria were randomized to 2 groups. Group A treated with systemic corticosteroids and antihistamines. Group B was treated with autologous haemotherapy. The efficacy of different therapeutic options, time of onset of disease and sex of newborns were assessed.

\section{Results}

Six caucasian pregnant women with a median age of 30 years (range 25 - 36) suffering from PEP were managed. In the majority of patients $(5 / 83 \%)$ PEP started in the third trimester. Only one patient $(17 \%)$ developed onset of the disease in the second trimester. The range of onset PEP varied from 19th to 37th week of pregnancy

Correspondence to: Martina Part MD, Faculty of Medicine, Comenius University in Bratislava, Dept. of Dermatology and Venerology, Mickiewiczova 13, 81369 Bratislava, Slovakia, Email -martina.part@icloud.com, 00421257290407

Key words: polymorphic eruption of pregnancy, pruritic urticarial papules and plaques of pregnancy, haemotherapy in pregnancy

Received: July 24, 2017; Accepted: August 14, 2017; Published: August 17, 2017 
(median 33 week). In all cases the eruption began in abdominal striae with progresion on extremities. Palms, face, soles and mucous membranes were not affected. The umbilical area was not involved. In two cases $(33 \%)$ targetoid lesions were presented. All others $(4 / 67$ $\%)$ presented with monomorphous urticarial eruption. Any additional disorders of pregnant women were not observed. Four women were primigravidae (67\%), two women (33\%) were secundigravidae. Their first pregnancy was without similar disorders. Group A (3/50 $\%)$ was treated with conservative therapeutic approach; systemic corticosteroids (40 mg prednison daily) and antihstamines (cetirizin or bisulepin perorally). This type of therapy revealed vague efficacy. Skin eruption was relativelly stable, regression of rash was not observed and pruritus was still reported. All patients in Group A use medication until delivery. In Group B we used alternative therapy with autologous haemotherapy. $10 \mathrm{ml}$ of venous blood was drawn from patient's vena cubitalis. Also, by intramuscular injections given in weekly intervals. Five $\mathrm{ml}$ of whole blood was immediately injected (new sterile needle was used) to each side of gluteal area. The injection of the whole blood was tolerated very well, any adverse reactions ( haematoma, abscess, bacterial infection, etc.) were not observed. Group B demonstrated remarkable improvment. Skin changes of PEP regressed after 10- 15 days. Patients underwent 1 or 2 cycles of intramuscular autologous haemotherapy depending on the clinical manifestaton and course of disease. Pruritus improved in 2 days after first dose in all cases. All patients (Group A + Group B) delivered healthy babies; five female babies $(83 \%)$ and in one case a male baby $(17 \%)$. Multiple gestation pregnancies were not recorded.

\section{Discussion}

The exact etiology of PEP remains unclear and likely it is multifactorial. There is no evidence associating to PEP with an autoimmune disease. Hormonal impact, dysbalance between immunological mechanisms during pregnancy, (decreased cellmediated imunity, predominacy of $\mathrm{T}_{\mathrm{H}} 2$ type specific switch from $\mathrm{T}_{\mathrm{H}} 1$ to $\mathrm{T}_{\mathrm{H}} 2$ immunity in placenta) and abnormal skin distension are suggested as causative agents of PEP [5]. Abdominal skin stretching can damage connective tissue, it results in release of collagen antigens which can trigger and activate immune system and inflammatory response [6]. The weight gain of the mother, and associated skin stretching is most apparent during the third trimester. This explains the manifestation of PEP with abdominal striae mainly in the third trimester. Mast cells physiologicaly influence pregnancy. However, the link between activation of mast cell and onset of PEP is speculated [7]. The activation of the skin immune system, increase of dendritic cells, and activated T- cells (6), high level of macrofages, eosinophils, and fetal DNA in skin infiltrates have been described in bioptic samples from affected skin features [2]. Activation of skin immune system [7]. includes predominantly activated T cells CD25, CD1a+ and CD54+. Maternal or fetal circulating antigens may be also responsible for rising of PEP [1]. It has not been established if there is also paternal contribution in development of PEP. Some authors believe that rising of PEP is due to paternal antigens presenting in the fetal part of placenta or due to placental products. In some studies PEP has been found with a reduced level of serum cortisol [3]. On the contrary in most studies there has not been found any difference found in women suffering from PEP versus healthy women [1]. The level of serum human chorionic gonadotropin, progesterone and estradiol is within normal range.

In general, the therapy of pregnant women is very challenging due to adverse events on fetus. The risk and benefit of treatment should be discussed in all cases. Conservative management with emollients, antipruritic topical agents, topical steroids, and oral antihistamines are used to diminish pruritus. In the past, systemic corticosteroids were recommended as a first line of therapy [8]. Oral corticosteroids can be given as middle doses (60-80 mg/day) [4]. There are some risks associated with oral corticosteroids, especially during first trimester. Increased risk of fracture, cataracts, suppression of the hypothalamo-pituitaryadrenal axis, flaring up of latent infections, production of peptic ulcer, and osteoporosis are reported complications [8]. Using corticosteroids as oral preparation during pregnancy can cause intrauterine growth restriction, maternal hyperglycemia, risk of sepsis, or high risk of oral cleft in the fetus. In refractory cases, we can use cyclosporine (2-3 mg/kg), especially where corticosteroids failed. Cyclosporine (CsA) is a fungal cyclic polypeptide with immunosuppressive effect. CsA is usually used in transplanted patients to protect rejection of a transplant [9]. In addition, this drug is used in the therapy of various autoimmune diseases such as inflammatory bowel disease, psoriasis, and rheumatoid arthritis. Unfortunately, CsA causes a lot of of side effects including neural toxicity, renal, hepatic, cardiac disorders. Kidney damage is the most frequent toxicity observed. According to a few reports, TNF alpha inhibitors can be safely used in patients with PEP [4]. The efficacy of various therapies is different but usually not very satisfying. The rash is still present. The patients suffer from severe itching, and sleeping disturbances. The pregnancy period may be alterated by the psychological state of the mother.

Autologous haemotherapy belongs to the old therapeutic procedures based on self-healing principles which result in benefical response of immune system. It was used in the therapy of chronic urticaria. Its efficacy in the reduction of disease activity and improvement in quality of life was shown in a randomized single blind study of chronic urticaria [10]. The autologous haemotherapy was also used for treatment of atopic dermatitis with the same efficacy [11]. We considered this type of therapy as a possible valuable treatment in our patiens with PEP. The exact mechanism of haemotherapy remains unclear. Immunomodulatory and self-healing effects are considered. It can probably activate cell mediated immune defence which can modulate reactivity of maternal immune system [5]. In our small study, we have recieved very similar results to the mentioned studies. We have achived regression of patologic skin changes and pruritus in very short period. The quality of life also improved.

\section{Conclusions}

The clinical presentation of PEP is variable. Diagnosis can be based on clinical polymorphic findings, because no specific laboratory or histological features have been confirmed. The therapy of polymorphic eruption of pregnancy is very limited, but it has tendency to spontaneous resolution after delivery. Emollients, topical or systemic corticosteroids and antihistamines are often used in the therapy of polymorphic eruption with different efficacy. Intramuscular autologous haemotherapy should be considered as an effective valuable therapeutic method with a very good efficacy and safety profile for mother as well as for the child. It is not an expensive procedure and it is very easy to perform. The great efficacy of autologous haemotherapy in PEP is documented in our study.

\section{Conflict of interest}

None

\section{Disclosure of funding}

None 


\section{References}

1. Brzoza Z, Kasperska-Zajac A, OleÅ` E, Rogala B (2007) Pruritic urticarial papules and plaques of pregnancy. J Midwifery Womens Health 52: 44-48. [Crossref]

2. Rudolph CM, Al-Fares S, Vaughan-Jones SA. et al. (2006) Polymorphic eruption of pregnancy: clinicopathology and potential trigger factors in 181 patients. Br J Dermatol 15: 54-60.

3. Ambros-Rudolph CM (2011) Dermatoses of pregnancy - clues to diagnosis, fetal risk and therapy. Ann Dermatol 23: 265-275. [Crossref]

4. Lehrhoff S, Pomeranz MK (2013) Specific dermatoses of pregnancy and their treatment. Dermatol Ther 26: 274-284. [Crossref]

5. Matz H, Orion E, Wolf R (2006) Pruritic urticarial papules and plaques of pregnancy: polymorphic eruption of pregnancy (PUPPP). Clin Dermatol 24: 105-108. [Crossref]

6. Soutou B, Aractingi S2 (2015) Skin disease in pregnancy. Best Pract Res Clin Obstet Gynaecol 29: 732-740. [Crossref]
7. Woidacki K, Zenclussen AC1, Siebenhaar F2 (2014) Mast cell-mediated and associated disorders in pregnancy: a risky game with an uncertain outcome? Front Immunol 5: 231. [Crossref]

8. Oren D, Nulman I, Makhija M, Ito S, Koren G (2004) Using corticosteroids during pregnancy. Are topical, inhaled, or systemic agents associated with risk? Can Fam Physician 50: 1083-1085. [Crossref]

9. Hagar HH (2004) The protective effect of taurine against cyclosporine A-induced oxidative stress and hepatotoxicity in rats. Toxicol Lett 151: 335-343

10. Kocaruk E., Aktas S,et al. (2012) Autologous whole blood and autologous serum injections are equally effective as placebo injections in reducing disease activity in patients with chronic spontaneous urticaria: a placebo controlled, randomized, singleblind study. J Dermatolog Treat 23: 465-71.

11. Pittler MH, Armstrong NC, Cox A et al. (2003) Randomized, double-blind, placebocontrolled trail of autologous blood therapy for atopic dermatitis. Br J Dermatol 148: 307-313.

Copyright: (C2017 Part M. This is an open-access article distributed under the terms of the Creative Commons Attribution License, which permits unrestricted use, distribution, and reproduction in any medium, provided the original author and source are credited. 